\title{
MicroRNA181a Is Overexpressed in T-Cell Leukemia/Lymphoma and Related to Chemoresistance
}

\author{
Zi-Xun Yan, ${ }^{1,2}$ Zhong Zheng, ${ }^{1,2}$ Wen Xue, ${ }^{1,2}$ Ming-Zhe Zhao, ${ }^{3}$ Xiao-Chun Fei, ${ }^{4}$ Li-Li Wu, ${ }^{4}$ \\ Li-Min Huang, ${ }^{5}$ Christophe Leboeuf, ${ }^{6}$ Anne Janin, ${ }^{6}$ Li Wang, ${ }^{1,2}$ and Wei-Li Zhao ${ }^{1,2}$ \\ ${ }^{1}$ State Key Laboratory of Medical Genomics, Shanghai Institute of Hematology, Shanghai Ruijin Hospital, School of Medicine, \\ Shanghai Jiao Tong University, Shanghai 200025, China \\ ${ }^{2}$ French-Chinese Laboratory of Genomic and Life Sciences, Laboratory of Molecular Pathology, Shanghai 200025, China \\ ${ }^{3}$ Department of Hematology, Central Hospital of Jinhua, Zhejiang 321000, China \\ ${ }^{4}$ Department of Pathology, Shanghai Ruijin Hospital, School of Medicine, Shanghai Jiao Tong University, Shanghai 200025, China \\ ${ }^{5}$ Department of Oncology, People's Hospital of Guizhou Province, Guiyang 550001, China \\ ${ }^{6}$ Laboratory of Pathology, Paris Diderot University, U1165 Inserm, 75010 Paris, France
}

Correspondence should be addressed to Li Wang; wl_wangdong@126.com and Wei-Li Zhao; zhao.weili@yahoo.com

Received 16 October 2014; Revised 20 January 2015; Accepted 27 January 2015

Academic Editor: Jin Wang

Copyright @ $2015 \mathrm{Zi}$-Xun Yan et al. This is an open access article distributed under the Creative Commons Attribution License, which permits unrestricted use, distribution, and reproduction in any medium, provided the original work is properly cited.

\begin{abstract}
MicroRNAs (miRs) play an important role in tumorogenesis and chemoresistance in lymphoid malignancies. Comparing with reactive hyperplasia, miR181a was overexpressed in 130 patients with T-cell leukemia/lymphoma, including acute T-cell lymphoblastic leukemia $(n=32)$, T-cell lymphoblastic lymphoma $(n=16)$, peripheral T-cell lymphoma, not otherwise specified $(n=45)$, anaplastic large cell lymphoma $(n=15)$, and angioimmunoblastic T-cell lymphoma $(n=22)$. Irrespective to histological subtypes, miR181a overexpression was associated with increased AKT phosphorylation. In vitro, ectopic expression of miR181a in HEK-293T cells significantly enhanced cell proliferation, activated AKT, and conferred cell resistance to doxorubicin. Meanwhile, miR181a expression was upregulated in Jurkat cells, along with AKT activation, during exposure to chemotherapeutic agents regularly applied to T-cell leukemia/lymphoma treatment, such as doxorubicin, cyclophosphamide, cytarabine, and cisplatin. Isogenic doxorubicin-resistant Jurkat and $\mathrm{H} 9$ cells were subsequently developed, which also presented with miR181a overexpression and cross-resistance to cyclophosphamide and cisplatin. Meanwhile, specific inhibition of miR181a enhanced Jurkat and H9 cell sensitivity to chemotherapeutic agents, further indicating that miR181a was involved in acquired chemoresistance. Collectively, miR181a functioned as a biomarker of T-cell leukemia/lymphoma through modulation of AKT pathway. Related to tumor cell chemoresistance, miR181a could be a potential therapeutic target in treating T-cell malignancies.
\end{abstract}

\section{Introduction}

Malignancies derived from the T-cell lineages encompass a heterogeneous group of neoplasm. The World Health Organization (WHO) classification recognizes distinctive subtypes of immature T-cell malignancies, like acute T-cell lymphoblastic leukemia (T-ALL) and T-cell lymphoblastic lymphoma (TLBL), as well as mature T-cell malignancies, mainly including peripheral T-cell lymphoma, not otherwise specified (PTCLNOS), anaplastic large cell lymphoma (ALCL), and angioimmunoblastic T-cell lymphoma (AITL) [1]. Varied from clinicopathological features and biological behavior, they are generally more aggressive than their B-cell counterpart, characterized by resistance to conventional chemotherapy and poor prognosis of the patients [2]. Therefore, biomarkers related to tumor progression and chemoresistance remain to be investigated and may become potential targets for future therapy in T-cell leukemia/lymphoma.

MicroRNAs (miRs), a class of 19- to 23-nucleotide noncoding RNA molecules, regulate gene expression by targeting mRNA at the $3^{\prime}$ untranslated region (UTR) [3]. Growing evidences suggested that miRs are critical regulators in tumorigenesis and drug resistance [4,5]. MiR181 is essential for lymphocyte differentiation and maturation in thymus [6]. 
More recently, it has been reported that miR181 overexpression promotes cell proliferation and activates PI3K/AKT signaling transduction pathway $[7,8]$. Activated in lymphoid malignancies [9], AKT plays a pivotal role in tumor progression and resistance to chemotherapeutic agents [10, 11]. Here we assessed miR181a expression, as well as its relation to AKT activation and chemoresistance in T-cell leukemia/lymphoma.

\section{Patients and Methods}

2.1. Patients. One hundred and thirty patients diagnosed with T-ALL or T-cell lymphoma were enrolled in this study, including 32 T-ALL, 16 T-LBL, 45 PTCL-NOS, 15 ALCL, and 22 AITL. Histologic diagnoses were established according to the WHO classification [1]. PTCL cases (PTCL-NOS, ALCL, and AITL) were treated with CHOP-based chemotherapy. T-LBL and T-ALL cases were treated with HyperCVAD$\mathrm{A} / \mathrm{B}$ regimens as previously reported $[12,13]$. Response rates were assessed according to the criteria as reported $[12,13]$. The clinicopathological data of the patients was listed in Table 1. Thirty-four age- and sex-matched cases with reactive hyperplasia were referred to as controls. The study was approved by the Institutional Review Board with informed consent obtained in accordance with the Declaration of Helsinki.

2.2. Cell Lines and Reagents. T-leukemia/lymphoma cell lines Jurkat, H9, and embryonic kidney cell line HEK-293T were available from American Type Culture Collection (Manassas, VA, USA). Doxorubicin-resistant Jurkat and H9 cells were established by exposure to gradually increasing concentrations of doxorubicin in vitro, as described by Huang et al. [14].

2.3. Cell Proliferation Assay. Cell proliferation was measured by MTT and EdU incorporation assay. Cells were seeded in 96-well plates and incubated with the indicated concentrations of reagents at $37^{\circ} \mathrm{C}$. After $72 \mathrm{~h}$ incubation, $0.1 \mathrm{mg}$ of MTT was added to each well and the absorbance was measured at $490 \mathrm{~nm}$ by spectrophotometry. EdU assay was conducted using Cell-Light EdU imaging kit (RiboBio, Guangzhou, China) according to the manufacturer's instruction.

2.4. MiR181a Detection. Total RNA was extracted from $20 \mu \mathrm{m}$ thick paraffin ( $n=100)$ or frozen sections $(n=64)$ using RecoverAll total nucleic acid isolation kit or Trizol agent following the manufacturer's protocol. MiR181a expression was analyzed by real-time quantitative RT-PCR using miRNA reverse transcription kit, hsa-miR 181a assay, and $7500 \mathrm{HT}$ Fast Real-time PCR system (Applied Biosystems, CA, USA). RNU24 was used as endogenous control and Jurkat cells for calibration. A relative quantification was calculated using the ${ }^{\Delta \Delta}$ CT method [15].

2.5. Western Blot. Cells were lysed in $200 \mu \mathrm{L}$ lysis buffer (0.5 M Tris- $\mathrm{HCl}, \mathrm{pH}$ 6.8, 2 mM EDTA, 10\% glycerol, $2 \%$ SDS, and $5 \% \beta$-mercaptoethanol). Protein extracts $(20 \mu \mathrm{g})$
TABLE 1: Clinicopathological characteristics of 130 patients with Tcell leukemia/lymphoma.

\begin{tabular}{|c|c|c|c|}
\hline Characteristics & $\begin{array}{l}\text { High miR18la } \\
\text { expression } \\
(n=65)\end{array}$ & $\begin{array}{c}\text { Low miR181a } \\
\text { expression } \\
(n=65)\end{array}$ & $P$ value \\
\hline \multicolumn{4}{|l|}{ Age (years) } \\
\hline$\geq 60$ & 13 & 17 & \multirow{2}{*}{0.5328} \\
\hline$<60$ & 52 & 48 & \\
\hline \multicolumn{4}{|l|}{ Gender } \\
\hline Male & 42 & 32 & \multirow{2}{*}{0.1106} \\
\hline Female & 23 & 33 & \\
\hline \multicolumn{4}{|l|}{$\begin{array}{l}\text { Lactic dehydrogenase } \\
\text { level (LDH) }\end{array}$} \\
\hline Normal & 19 & 23 & \multirow{2}{*}{0.5740} \\
\hline Above normal & 46 & 42 & \\
\hline \multicolumn{4}{|l|}{ Pathological subtypes } \\
\hline T-ALL & 15 & 17 & \multirow{5}{*}{0.6656} \\
\hline T-LBL & 8 & 8 & \\
\hline PTCL-NOS & 22 & 23 & \\
\hline ALCL & 6 & 9 & \\
\hline AITL & 14 & 8 & \\
\hline \multicolumn{4}{|l|}{$\begin{array}{l}\text { International } \\
\text { prognostic index (IPI) }\end{array}$} \\
\hline Low & 12 & 11 & \multirow{4}{*}{0.3152} \\
\hline Low/intermediate & 23 & 33 & \\
\hline Intermediate/high & 20 & 15 & \\
\hline High & 10 & 6 & \\
\hline $\begin{array}{l}\text { Overall response }(\mathrm{CR} \\
+\mathrm{PR})\end{array}$ & 38 & 50 & 0.0385 \\
\hline
\end{tabular}

were electrophoresed on $10 \%$ SDS polyacrylamide gels and transferred to nitrocellulose membranes. Membranes were blocked with 5\% nonfat dried milk in Tris-buffered saline and incubated for $2 \mathrm{~h}$ at room temperature with appropriate primary antibody, followed by horseradish peroxidaseconjugated secondary antibody. The immunocomplexes were visualized using chemiluminescence phototope-horseradishperoxidase kit. Actin was used to ensure equivalent protein loading. Antibodies against phosphorylated-AKT (p-AKT), AKT, actin, and chemiluminescence phototope-horseradishperoxidase kit were obtained from Cell Signaling (Beverly, MA, USA). Anti-PTEN antibody was from Abcam (Cambridge, UK). Horseradish peroxidase-conjugated goat antimouse-IgG and goat anti-rabbit-IgG antibodies were from Santa Cruz Biotechnology (Santa Cruz, CA, USA).

2.6. Cell Transfection. HEK-293T cells were incubated with pEZX-181a vector (HmiR0292-MR03) or a control vector pEZX-ct (CmiR0001-MR03, Genecopia, MD, USA) and Li pofectamine 2000 (Invitrogen, CA, USA) for $24 \mathrm{~h}$ and replaced in fresh medium for further experiments. To inhibit miR181a expression, Jurkat and H9 cells were transfected with $10 \mathrm{nM}$ antagomir using Lipofectamine 2000 (Invitrogen) for 


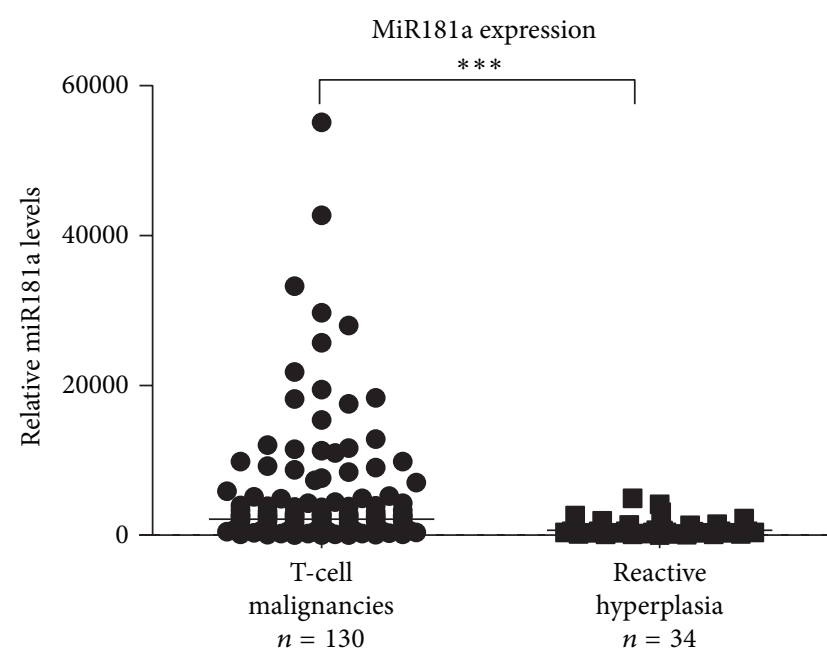

(a)

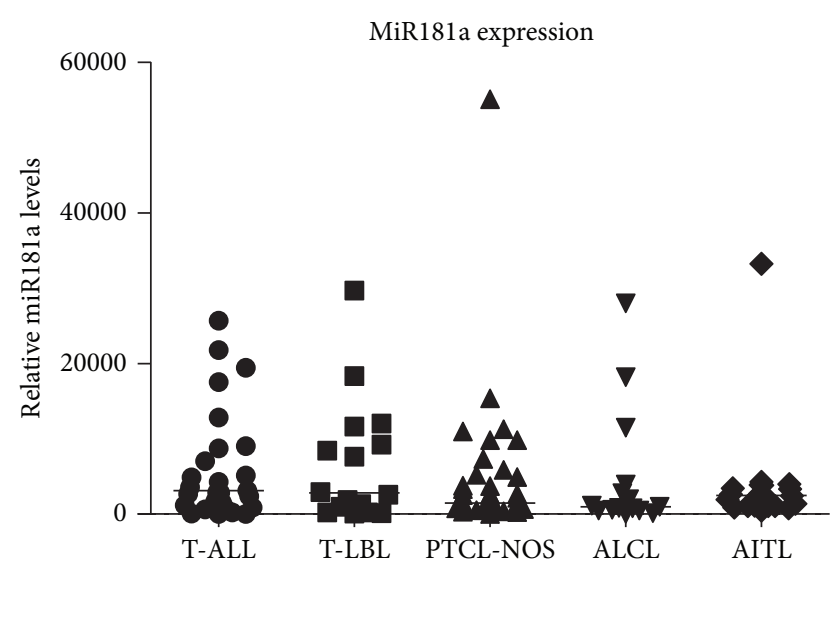

(b)

FIgURE 1: MiR181a was overexpressed in T-cell leukemia/lymphoma. (a) As detected by real-time quantitative PCR, miR181a was overexpressed in T-cell malignancies. ${ }^{* * *} P<0.001$ comparing with reactive hyperplasia. The relative expression level of each patient was calculated based on the lowest expression value. (b) MiR181a was overexpressed in acute T-cell lymphoblastic leukemia (T-ALL, $n=32$ ), as well as subtypes of T-cell lymphoma, including T-cell lymphoblastic lymphoma (T-LBL, $n=16$ ), peripheral T-cell lymphoma, not otherwise specified (PTCL-NOS, $n=45$ ), anaplastic large cell lymphoma (ALCL, $n=15$ ), and angioimmunoblastic T-cell lymphoma (AITL, $n=22$ ).

$24 \mathrm{~h}$. The miR181a antagomir and the negative control were synthesized by Shanghai Biotend Biotechnologies Co., Ltd. (Shanghai, China).

2.7. Immunohistochemistry Assay. Immunohistochemistry was performed on $5 \mu \mathrm{m}$ paraffin sections with an indirect immunoperoxidase method using antibodies against $\mathrm{p}$-AKT (Cell Signaling). Expression levels were scored semiquantitatively based on percentage of positive cells:,$+<25 \%$; ++ , 25-49\%; +++, 50-74\%; ++++, 75-100\%.

2.8. Statistical Analysis. Differences of miR181a expression among groups were assessed using Mann-Whitney $U$ test. The association between miR181a and p-AKT expression in human tumor samples was analyzed by Fisher's exact test. In vitro experimental results were expressed as mean \pm S.D. of data obtained from three separate experiments and determined using $t$-test to compare variance. $P<0.05$ was considered statistically significant.

\section{Results}

3.1. MiR181a Was Overexpressed in T-Cell Leukemia/Lymphoma and Related to AKT Activation. Compared with reactive hyperplasia, miR181a was overexpressed in T-cell leukemia/ lymphoma $(P<0.0001$, Figure $1(\mathrm{a}))$. No significant difference was observed among T-ALL and subtypes of T-cell lymphoma $(P=0.5153$, Figure $1(\mathrm{~b}))$.

The median value of relative miR181a expression in Tcell leukemia/lymphoma was 2136 . The patients with miR181a expression level over and equal to the median value were regarded as high miR181a expression, whereas those below the median value were included in the low miR181a expression. Patients with high miR181a expression had significantly lower overall response rate (ORR) than those with low miR181a expression (Table 1). P-AKT expression was detected by immunohistochemistry in primary tumor sections of 12 T-cell lymphoma patients (6 cases from high miR181a expression group and 6 cases from low miR18la expression group, Figure 2(a)). High miR181a expression was associated with increased positivity of p-AKT $(P=0.0152$, Figure $2(b))$.

3.2. MiR181a Promoted Cell Proliferation and Induced Chemoresistance through Activating AKT. T-leukemia/lymphoma cell lines Jurkat and H9 possessed higher levels of miR181a expression than that of HEK-293T cells $(P=0.0023$ and $P=$ 0.0030 , resp., Figure 3(a)). To gain insight into the biological function of miR181a, HEK-293T cells, with lowest miR181a expression, were transiently transfected with miR181a (pEZX181a, Figure 3(b)). Ectopic expression of miR181a remarkably accelerated cell growth, as compared to the control cells (pEZX-ct). In parallel with increased cell proliferation, the percentage of EdU-positive cells was significantly higher in pEZX-181a cells $(52.7 \% \pm 8.7 \%)$ than in pEZX-ct cells $(20.7 \% \pm$ $7.0 \%, P=0.0458$, Figure 3(c)). Of note, overexpression of miR181a increased AKT phosphorylation, while the total protein level remained constant (Figure 3(d)). AKT is the key regulator of cell proliferation and drug resistance [11, 16]. Accordingly, the IC50 of doxorubicin was significantly increased in the miR181a-overexpressing HEK-293T cells, as compared to the control cells $(21.3 \pm 3.1 \mathrm{nM}$ versus $9.8 \pm$ $2.3 \mathrm{nM}, P=0.0260$, Figure 3(e)).

3.3. MiR181a Overexpression Corresponded to Chemoresistance in T-Leukemia/Lymphoma Cells. Doxorubicin (DOX), 


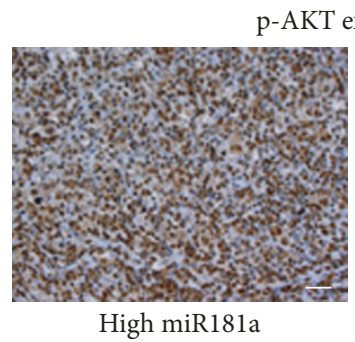

High miR181a $n=6$

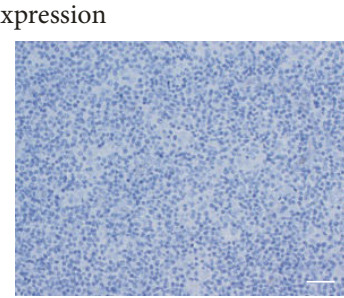

Low miR181a $n=6$

(a)

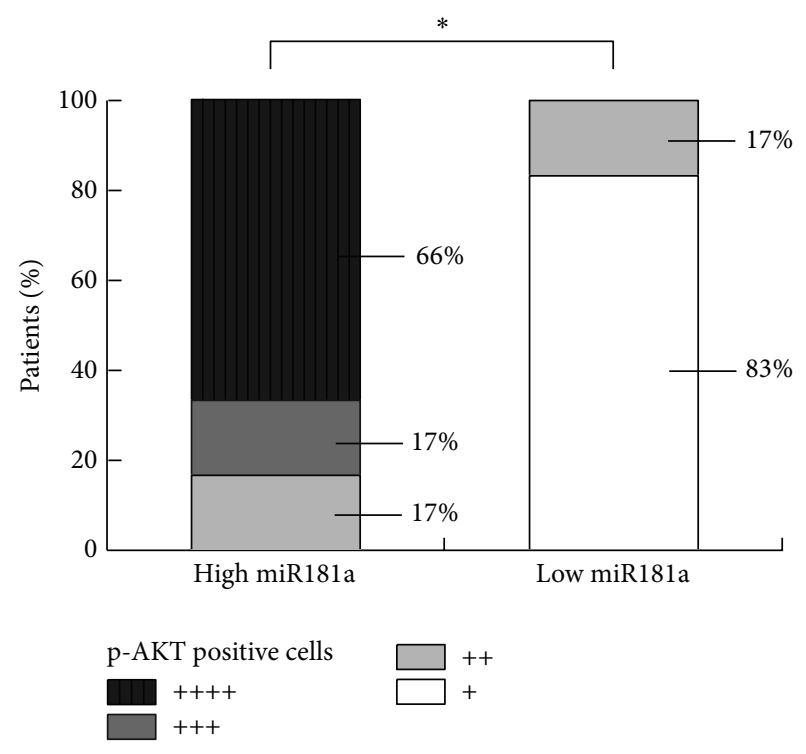

(b)

FIGURE 2: MiR181a overexpression was related to AKT activation in T-cell leukemia/lymphoma. As revealed by immunohistochemistry (a), increased positivity of p-AKT was observed in primary tumor samples of T-cell lymphoma patients with high miR181a expression $(n=6)$, compared to those with low miR181a expression $(n=6)(\mathrm{b}) .{ }^{*} P<0.05$ comparing with low miR181a expression. Bar $=50 \mu \mathrm{m}$.

cyclophosphamide (CTX), cytarabine (Ara-C), and cisplatin are main chemotherapeutic agents used in treating T-cell malignancies. When Jurkat cells were exposed to these agents for $48 \mathrm{~h}$, miR181a expression was significantly increased $(P=$ 0.0019, $P=0.0016, P=0.0172$, and $P<0.0001$, resp., Figure 4(a)), in accordance with increased AKT phosphorylation detected by Western blot (Figure 4(b)).

Acquired drug resistance is an important obstacle that impairs the success of cancer treatment. Isogenic doxorubicin-resistant sublines were developed as previously reported $[14,17]$ at the concentrations of $7.5 \mathrm{nM}$ (Jurkat/7.5 nM DOX) and $15 \mathrm{nM}$ (Jurkat/15 nM DOX) in Jurkat cells, as well as $5 \mathrm{nM}$ (H9/5 nM DOX) and $10 \mathrm{nM}(\mathrm{H} 9 / 10 \mathrm{nM}$ DOX) in H9 cells. Compared with the parental cells (Jurkat cells, $44.3 \pm 4.0 \mathrm{nM}$; H9 cells, $14.7 \pm 5.5 \mathrm{nM}$ ), IC50 of doxorubicin was significantly increased in Jurkat/7.5 nM DOX and Jurkat/ $15 \mathrm{nM}$ DOX cells $(70.1 \pm 8.0 \mathrm{nM}$ and $100.0 \pm 8.0 \mathrm{nM}, P=0.0453$ and $P=0.0152$, resp., Figure 4(c)) and in H9/5 nM DOX and H9/10 nM DOX cells $(28.0 \pm 4.3 \mathrm{nM}$ and $41.3 \pm 4.7 \mathrm{nM}, P=0.0481$ and $P=0.0443$, resp., Figure 4(d)). Accordingly, levels of miR181a expression were significantly higher in doxorubicin-resistant cells than in the parental cells $(1.8 \pm 0.2$-fold in Jurkat $/ 7.5 \mathrm{nM}$ DOX and 2.8 \pm 0.3 -fold in Jurkat $/ 15 \mathrm{nM}$ DOX cells, $P=0.0253$ and $P=0.0112$, Figure $4(\mathrm{e}) ; 2.1 \pm 0.6$-fold in $\mathrm{H} 9 / 5 \mathrm{nM}$ and $3.4 \pm 0.2$-fold in H9/10 nM DOX cells, $P=0.0384$ and $P=$ 0.0032, resp., Figure 4(f)), consistent with AKT activation (Figures $4(\mathrm{~g})$ and $4(\mathrm{~h})$ ). Increased miR181a expression was linked to AKT phosphorylation, not only in PTEN-negative Jurkat cells [18] but also in PTEN-positive H9 cells (Figures $4(\mathrm{~g})$ and $4(\mathrm{~h})$ ). Therefore, regulation of AKT phosphorylation could be independent of PTEN expression in T-cell leukemia/lymphoma. Further drug-sensitivity test showed that these resistant sublines with miR181a overexpression also had cross-resistance to other chemotherapeutic agents (Table 2). Therefore, exposure to chemotherapeutic agents could induce miR181a expression and AKT activation, which is closely related to acquired chemoresistance.

3.4. MiR181a Inhibition Enhanced T-Leukemia/Lymphoma Cell Sensitivity to Chemotherapeutic Agents. Specific inhibition of miR181a in Jurkat and H9 cells as well as their resistant sublines, using an antagomir, significantly increased cell sensitivity to doxorubicin (Figures 5(a) and 5(b)) and decreased AKT phosphorylation (Figures 5(c) and 5(d)). Similar to doxorubicin, decreased miR181a expression was also related to reduced IC50 of cisplatin and cyclophosphamide in doxorubicin-resistant Jurkat and H9 cells (Table 3).

\section{Discussion}

In addition to genetic abnormalities, epigenetic aberrations, particularly those of miRs, participate in human carcinogenesis $[5,19]$. MiR181a is critically involved in hematological malignancies. In acute myelogenous leukemia, increased miR181a expression was significantly associated with a higher complete remission rate of the patients [20]. However, as recently reported in lymphoid malignancies like acute lymphoblastic leukemia and multiple myeloma, miR181 overexpression was related to advanced stage and tumor progression $[7,21]$. In accordance with malignant $\mathrm{T}$ cells, miR181a is 


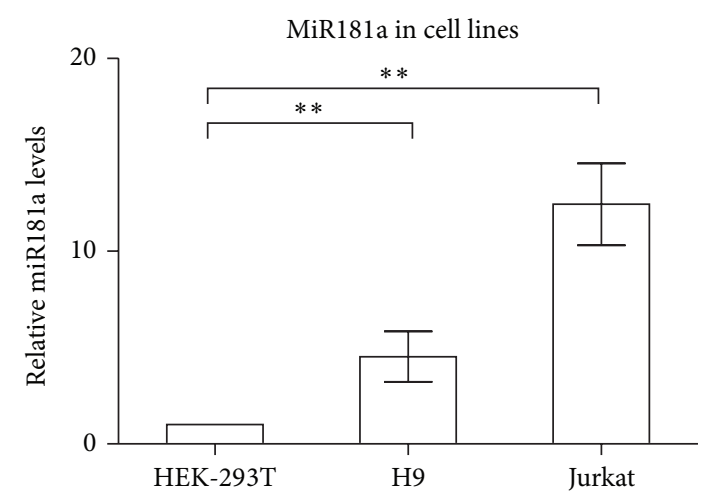

(a)
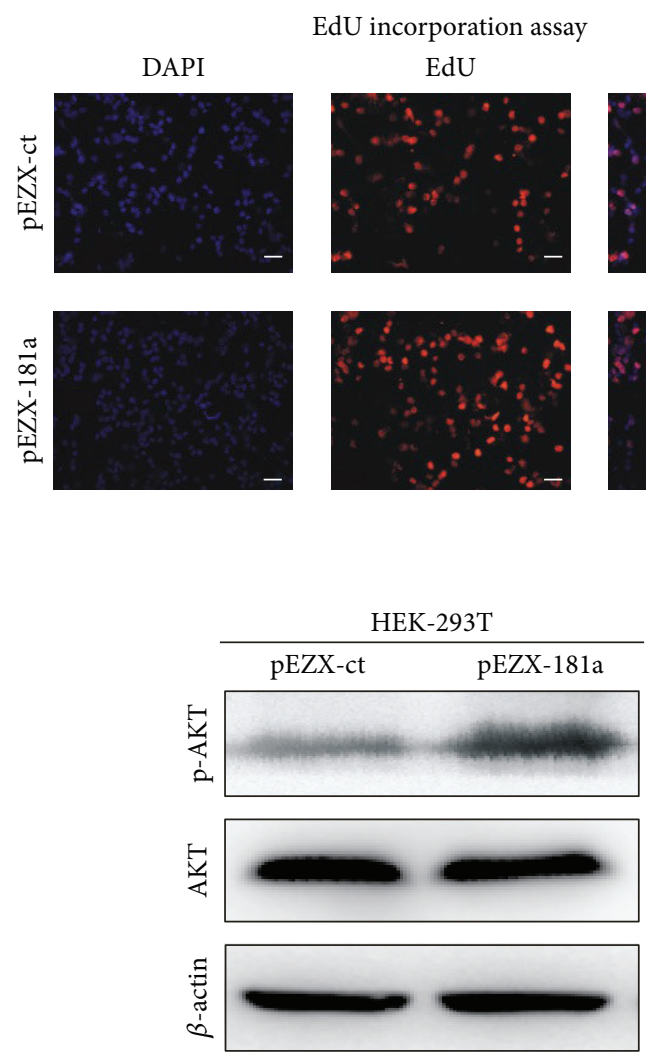

(d)

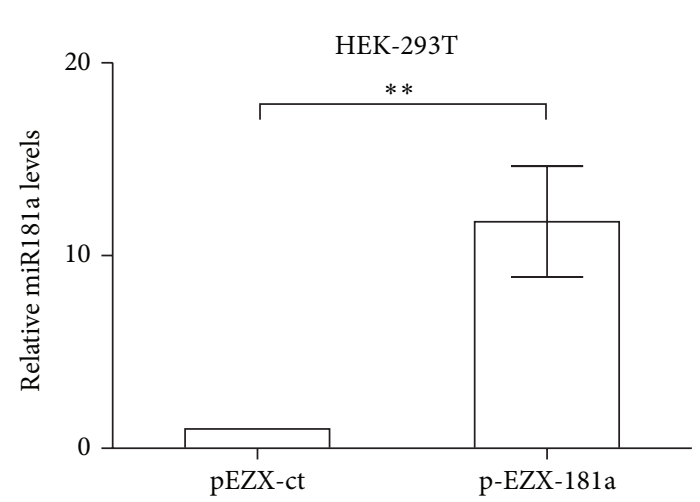

(b)

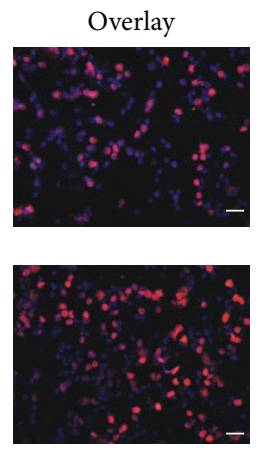

(c)

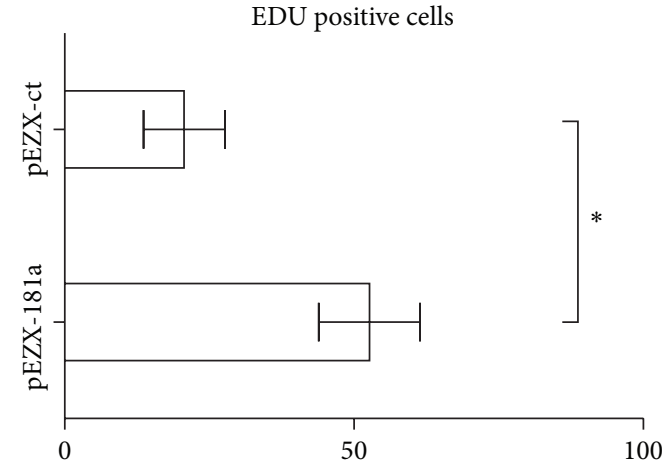

(\%)

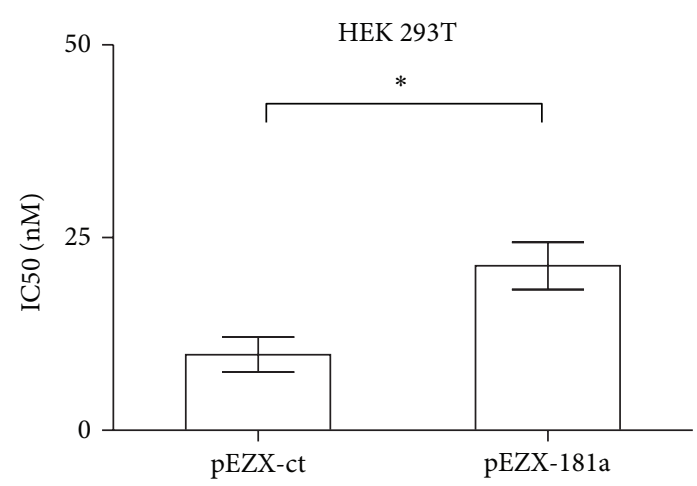

(e)

FIGURE 3: Ectopic expression of miR181a enhanced cell proliferation and resistance to doxorubicin through AKT activation. (a) Tleukemia/lymphoma Jurkat and H9 cells had significantly higher expression levels of miR181a than that of HEK-293T cells. ${ }^{* *} P<0.01$ comparing with HEK-293T cells. (b) Transfection with miR181a (pEZX-181a) in HEK-293T cells resulted in significantly increased miR181a expression. ${ }^{* *} P<0.01$ comparing with the control pEZX-ct cells. (c) EdU incorporation assay in HEK-293T cells showed that miR181aoverexpressing pEZX-181a cells presented with increased EdU-positive cells. ${ }^{*} P<0.05$, comparing with the control pEZX-ct cells. Bar $=$ $20 \mu \mathrm{m}$. (d) Overexpression of miR181a increased AKT phosphorylation, while the total protein level remained constant. (e) IC50 of doxorubicin was significantly higher in the pEZX-181a cells than in the control pEZX-ct cells. ${ }^{*} P<0.05$ comparing with the control pEZX-ct cells.

upregulated in normal T-cell counterpart and miR181a/bdeficient mice show severe defects in T-cell development $[6,8]$. Therefore, miR181 may have different roles in hematological malignancies. Our study showed that miR181a, independent of histological subtypes, was overexpressed and these patients were less responding to treatment, referring to
miR181a as a common biomarker of chemoresistance in T-cell leukemia/lymphoma.

Chemoresistance, determining therapeutic effect and clinical outcome of the patients, is one of the control factors in cancer treatment, including T-cell leukemia/lymphoma [22]. Here miR181a was closely related to chemoresistance 


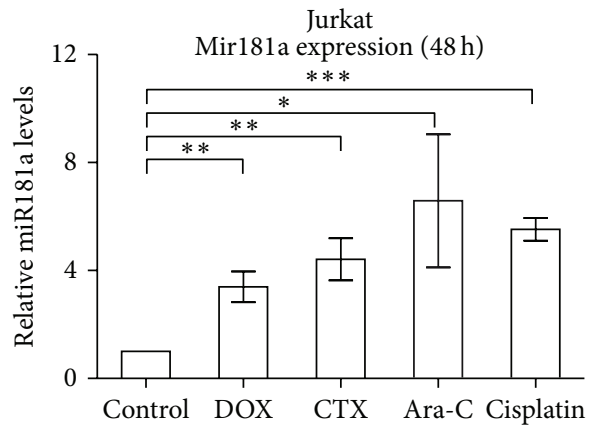

(a)

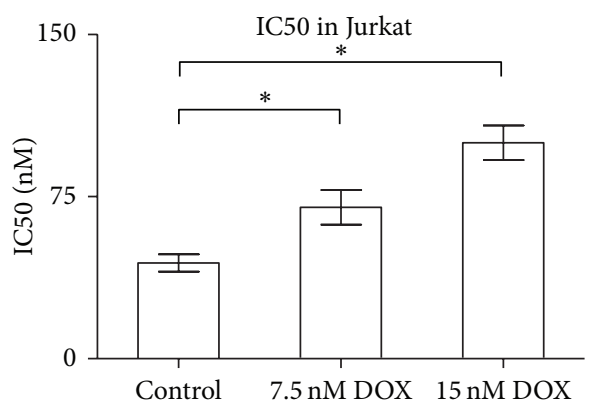

(c)

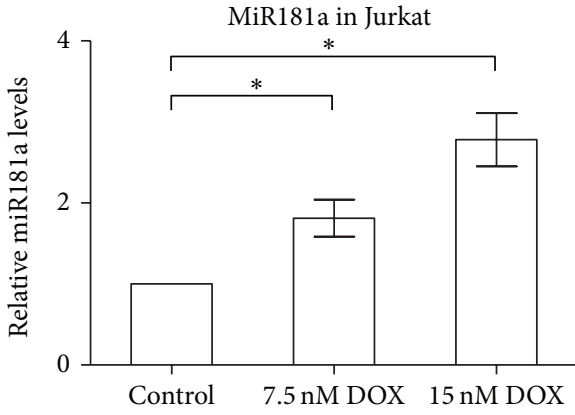

(e)

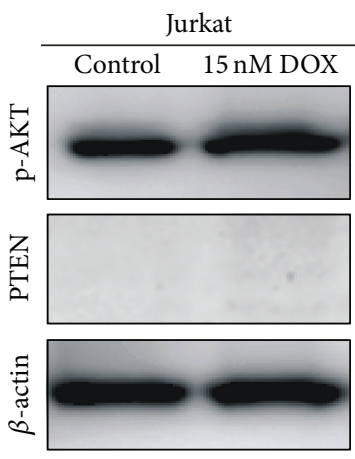

(g)

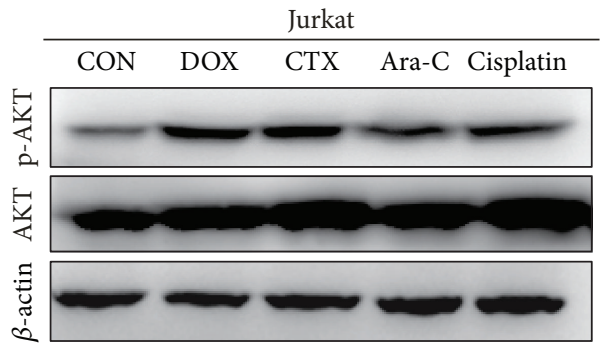

(b)

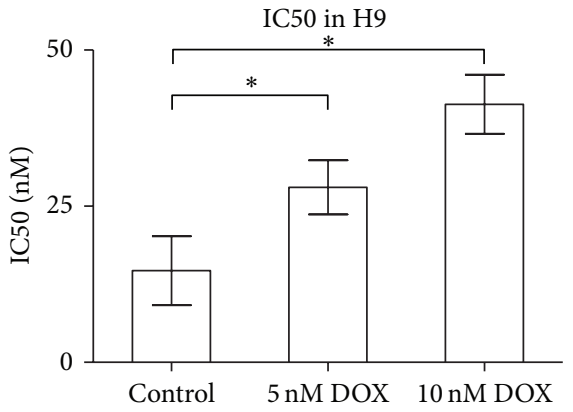

(d)

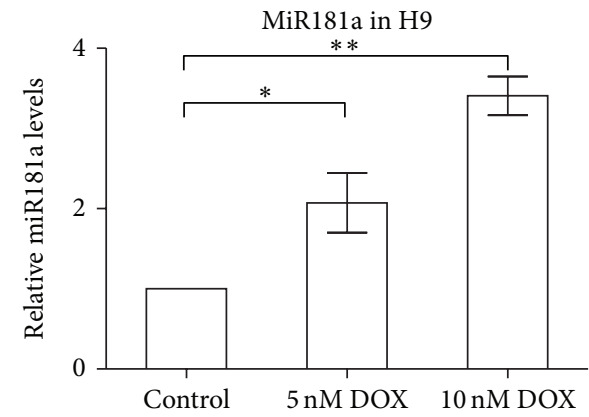

(f)

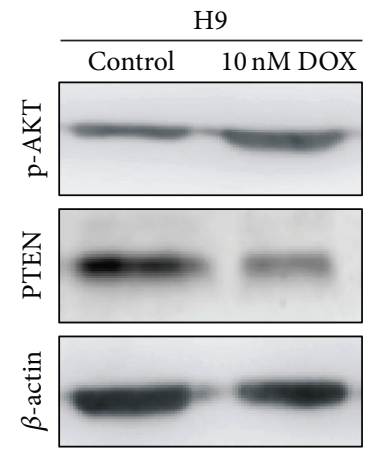

(h)

FIGURE 4: Exposure of T-leukemia/lymphoma cells to chemotherapeutic agents upregulated miR181a expression and resulted in AKT activation. (a) When Jurkat cells were treated with chemotherapeutic agents for $48 \mathrm{~h}$, miR181a expression was significantly increased. CON, untreated; DOX, doxorubicin; CTX, cyclophosphamide; Ara-C, cytarabine. ${ }^{*} P<0.05$; ${ }^{* *} P<0.01$; ${ }^{* * *} P<0.001$ comparing with the CON cells. (b) In accordance with miR181a upregulation, increased AKT phosphorylation was observed by western blot, while the total protein level remained constant. (c) and (d) IC50 of doxorubicin was significantly increased in doxorubicin-resistant Jurkat (c) and H9 (d) cells, which were exposed to doxorubicin for 3 weeks. (e) and (f) MiR181a expression was significantly increased in doxorubicin-resistant Jurkat (e) and $\mathrm{H} 9$ (f) cells. CON, untreated; ${ }^{*} P \leq 0.05 ;{ }^{* *} P \leq 0.01$ comparing with the CON cells. (g) and (h) P-AKT expression was significantly increased in doxorubicin-resistant Jurkat (g) and H9 (h) cells. 
TABLE 2: Cross-resistance of doxorubicin-resistant cells to other chemotherapeutic agents.

\begin{tabular}{|c|c|c|c|c|c|c|}
\hline \multirow[b]{2}{*}{ Agents } & \multicolumn{3}{|c|}{ IC50 } & \multicolumn{2}{|c|}{ IC50 } & \multirow[b]{2}{*}{$P$ value } \\
\hline & Jurkat & $\begin{array}{c}\text { Doxorubicin-resistant } \\
\text { Jurkat }\end{array}$ & $P$ value & $\mathrm{H} 9$ & $\begin{array}{c}\text { Doxorubicin- } \\
\text { resistant } \\
\text { H9 }\end{array}$ & \\
\hline Cisplatin (uM) & $6.2 \pm 0.3$ & $9.0 \pm 0.4$ & 0.0039 & $5.7 \pm 0.3$ & $8.5 \pm 0.5$ & 0.0067 \\
\hline Cyclophosphamide (mM) & $3.3 \pm 0.2$ & $4.8 \pm 0.2$ & 0.0056 & $2.4 \pm 0.2$ & $3.9 \pm 0.3$ & 0.0106 \\
\hline
\end{tabular}

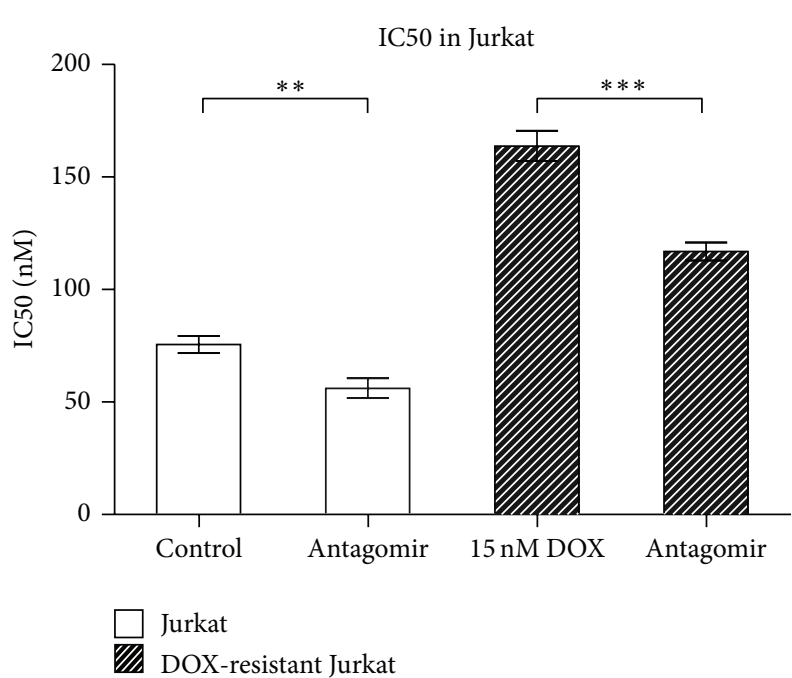

(a)

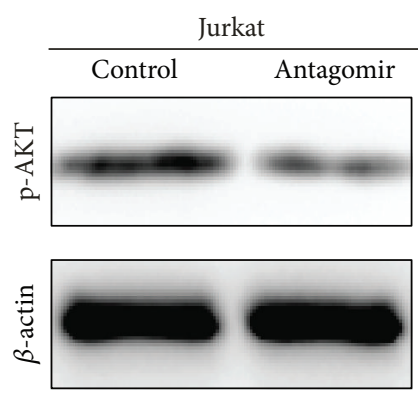

(c)

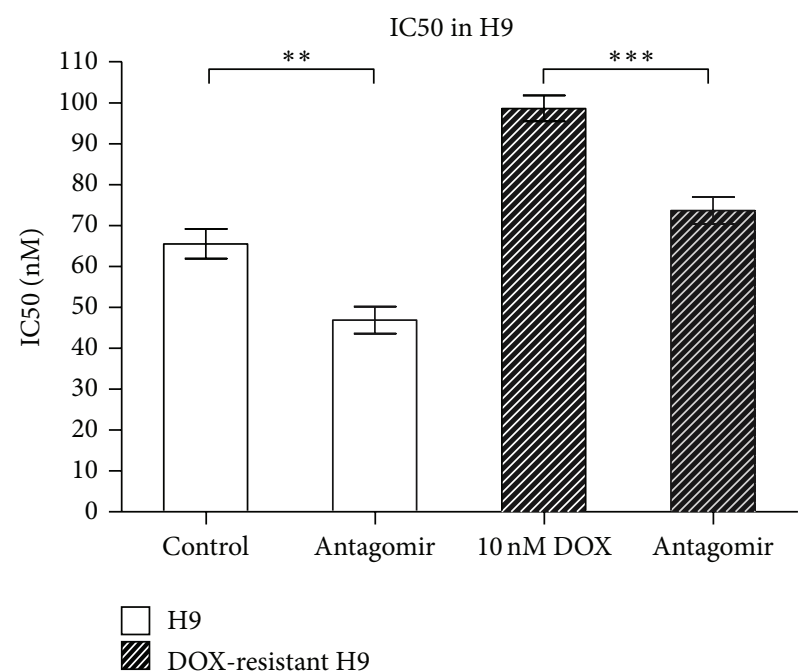

(b)

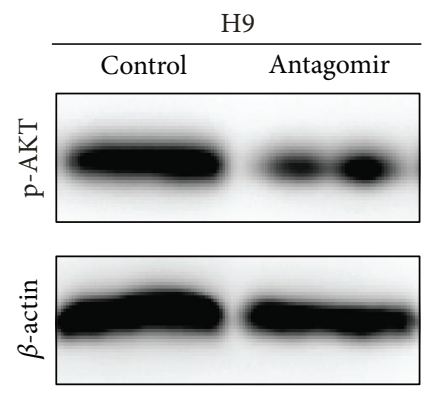

(d)

FIGURE 5: Specific inhibition of miR181a expression could increase T-leukemia/lymphoma cells and resistant sublines sensitivity to doxorubicin, along with p-AKT downregulation. (a) and (b) IC50 of doxorubicin was significantly decreased in antagomir-treated Jurkat (a) and H9 (b) cells. The DOX-resistant Jurkat and H9 cells were exposed to doxorubicin for 6 weeks. ${ }^{* *} P \leq 0.01$; ${ }^{* * *} P \leq 0.001$ comparing with the control cells. (c) and (d) Decreased AKT phosphorylation was found in miR18la antagomir-treated Jurkat (c) and H9 (d) cells.

in T-leukemia/lymphoma. This was consistent with previous reports in B-cell lymphoma that high expression of miR181 could lead to decreasing proapoptotic protein Bim and increasing resistance to chemotherapy [23]. AKT is a key tuning point in tumor cell growth and chemosensitivity $[24,25]$. MiR181 is a central regulator of PI3K pathway, since miR181a/b-deficient mice showed severe defects in lymphoid development and T-cell homeostasis associated with impaired PI3K/AKT cascade [8]. As mechanism of action, miR181 targets PTPN, DUSP5, and DUSP6, resulting in $\mathrm{PI}$ K/AKT activation and tumorigenesis in murine T-cell leukemia $[7,8]$. Our results showed that ectopic expression of miR181a leads to AKT phosphorylation, enhancing cell proliferation and inducing cell resistance to chemotherapy in T-cell leukemia/lymphoma. This correlation of miR181a overexpression with AKT activation was observed not only in cell lines but also in primary tumor samples of patients with Tleukemia/lymphoma. Apart from primary chemoresistance, acquired chemoresistance is also an important factor of treatment failure. MiR181a expression of Jurkat cells was significantly upregulated after exposure to chemotherapeutic agents and linked to increased AKT phosphorylation. Meanwhile, 


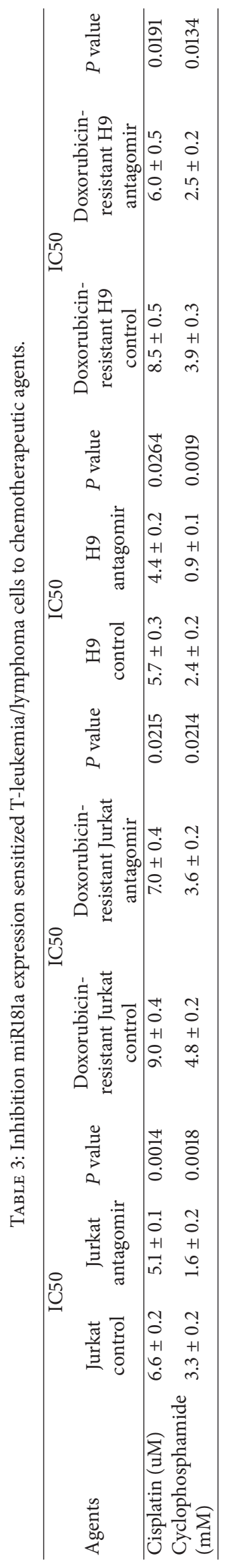


isogenic doxorubicin-resistant cell lines were developed, which were resistant to doxorubicin and had cross-resistance to other chemotherapeutic drugs. The relative resistance to chemotherapeutic agents was along with increased miR181a expression and subsequent AKT activation, further confirming that miR181a induced AKT activation and contributed to chemoresistance in T-cell leukemia/lymphoma.

\section{Conclusions}

MiR181a was involved in T-cell leukemia/lymphoma through modulation of AKT pathway. Functioned as a critical regulator of chemosensitivity, miR181a could thus be a promising therapeutic target in treating T-cell malignancies resistant to chemotherapy.

\section{Conflict of Interests}

The authors declare that there is no conflict of interests regarding the publication of this paper.

\section{Authors' Contribution}

Zi-Xun Yan, Zhong Zheng, Wen Xue, and Ming-Zhe Zhao contributed equally to this paper.

\section{Acknowledgments}

This work was supported, in part, by the National Natural Science Foundation of China (81172254, 81101793, and 81325003), Innovation Fund Projects of Shanghai Jiao Tong University (BXJ201312), the Shanghai Commission of Science and Technology (11JC1407300, 14430723400, and 14140903100), and the Program of Shanghai Subject Chief Scientists (13XD1402700).

\section{References}

[1] E. S. Jaffe, "The 2008 WHO classification of lymphomas: implications for clinical practice and translational research," Hematology/the Education Program of the American Society of Hematology, pp. 523-531, 2009.

[2] W.-L. Zhao, "Targeted therapy in T-cell malignancies: dysregulation of the cellular signaling pathways," Leukemia, vol. 24, no. 1, pp. 13-21, 2010.

[3] D. Lee and C. Shin, "MicroRNA-target interactions: new insights from genome-wide approaches," Annals of the New York Academy of Sciences, vol. 1271, no. 1, pp. 118-128, 2012.

[4] J. W. Kim, S. Mori, and J. R. Nevins, "Myc-induced microRNAs integrate Myc-mediated cell proliferation and cell fate," Cancer Research, vol. 70, no. 12, pp. 4820-4828, 2010.

[5] H. Y. Jin, H. Oda, M. Lai et al., "MicroRNA-17 92 plays a causative role in lymphomagenesis by coordinating multiple oncogenic pathways," The EMBO Journal, vol. 32, no. 17, pp. 23772391, 2013.

[6] C.-Z. Chen, L. Li, H. F. Lodish, and D. P. Bartel, "MicroRNAs modulate hematopoietic lineage differentiation," Science, vol. 303, no. 5654, pp. 83-86, 2004.
[7] R. Fragoso, T. Mao, S. Wang et al., "Modulating the strength and threshold of NOTCH oncogenic signals by mir-181a-1/b-1," PLoS Genetics, vol. 8, no. 8, Article ID e1002855, 2012.

[8] J. Henao-Mejia, A. Williams, L. Goff et al., "The microRNA miR-181 is a critical cellular metabolic rheostat essential for NKT cell ontogenesis and lymphocyte development and homeostasis," Immunity, vol. 38, no. 5, pp. 984-997, 2013.

[9] W.-Y. Shi, D. Xiao, L. Wang et al., "Therapeutic metformin/ AMPK activation blocked lymphoma cell growth via inhibition of mTOR pathway and induction of autophagy," Cell Death and Disease, vol. 3, article e275, 2012.

[10] K.-F. Chen, H.-L. Chen, W.-T. Tai et al., "Activation of phosphatidylinositol 3-kinase/Akt signaling pathway mediates acquired resistance to sorafenib in hepatocellular carcinoma cells," Journal of Pharmacology and Experimental Therapeutics, vol. 337, no. 1, pp. 155-161, 2011.

[11] C. Knuefermann, Y. Lu, B. Liu et al., "HER2/PI-3K/Akt activation leads to a multidrug resistance in human breast adenocarcinoma cells," Oncogene, vol. 22, no. 21, pp. 3205-3212, 2003.

[12] H. M. Kantarjian, S. O’Brien, T. L. Smith et al., "Results of treatment with hyper-CVAD, a dose-intensive regimen, in adult acute lymphocytic leukemia," Journal of Clinical Oncology, vol. 18, no. 3, pp. 547-561, 2000.

[13] D. A. Thomas, S. O'Brien, J. Cortes et al., "Outcome with the hyper-CVAD regimens in lymphoblastic lymphoma," Blood, vol. 104, no. 6, pp. 1624-1630, 2004.

[14] L. Huang, C. Perrault, J. Coelho-Martins et al., "Induction of acquired drug resistance in endothelial cells and its involvement in anticancer therapy," Journal of Hematology and Oncology, vol. 6, article 49, 2013.

[15] D.-J. Min, T. Ezponda, M. K. Kim et al., "MMSET stimulates myeloma cell growth through microRNA-mediated modulation of c-MYC," Leukemia, vol. 27, no. 3, pp. 686-694, 2013.

[16] X. Zhu, Z. He, J. Wu et al., "A marine anthraquinone SZ-685C overrides adriamycin-resistance in breast cancer cells through suppressing Akt signaling," Marine Drugs, vol. 10, no. 4, pp. 694-711, 2012.

[17] W.-J. Li, S.-L. Zhong, Y.-J. Wu et al., "Systematic expression analysis of genes related to multidrugresistance in isogenic docetaxel-and adriamycin-resistant breast cancer cell lines," Molecular Biology Reports, vol. 40, no. 11, pp. 6143-6150, 2013.

[18] M. Freeley, J. Park, K. J. Yang et al., "Loss of PTEN expression does not contribute to PDK-1 activity and PKC activationloop phosphorylation in Jurkat leukaemic T cells," Cellular Signalling, vol. 19, no. 12, pp. 2444-2457, 2007.

[19] Z.-X. Yan, L.-L. Wu, K. Xue et al., "MicroRNA187 overexpression is related to tumor progression and determines sensitivity to bortezomib in peripheral T-cell lymphoma," Leukemia, vol. 28, no. 4, pp. 880-887, 2014.

[20] S. Schwind, K. Maharry, M. D. Radmacher et al., "Prognostic significance of expression of a single microRNA, miR-181a, in cytogenetically normal acute myeloid leukemia: a cancer and leukemia group B study," Journal of Clinical Oncology, vol. 28, no. 36, pp. 5257-5264, 2010.

[21] C. Bi and W. J. Chng, "MicroRNA: important player in the pathobiology of multiple myeloma," BioMed Research International, vol. 2014, Article ID 521586, 12 pages, 2014.

[22] W. L. Zhao, "Targeted therapy in T-cell malignancies: dysregulation of the cellular signaling pathways," Leukemia, vol. 24, no. 1, pp. 13-21, 2010. 
[23] T. Lwin, J. Lin, Y. S. Choi et al., "Follicular dendritic celldependent drug resistance of non-Hodgkin lymphoma involves cell adhesion-mediated Bim down-regulation through induction of microRNA-181a," Blood, vol. 116, no. 24, pp. 5228-5236, 2010.

[24] L. S. Steelman, S. L. Abrams, J. Whelan et al., "Contributions of the Raf/MEK/ERK, PI3K/PTEN/Akt/mTOR and Jak/STAT pathways to leukemia," Leukemia, vol. 22, no. 4, pp. 686-707, 2008.

[25] D. A. Fruman and C. Rommel, "PI3K and cancer: lessons, challenges and opportunities," Nature Reviews Drug Discovery, vol. 13, no. 2, pp. 140-156, 2014. 


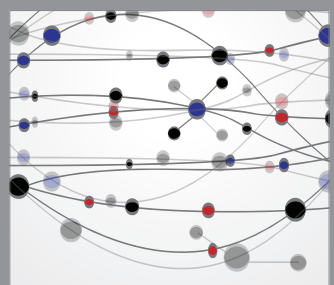

The Scientific World Journal
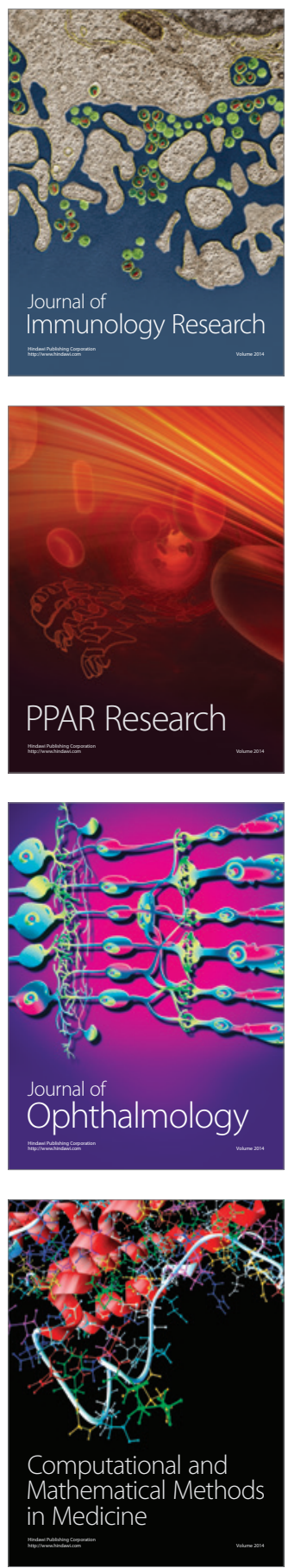

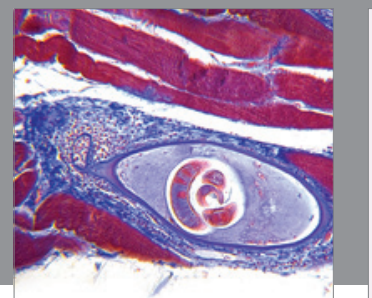

Gastroenterology

Research and Practice
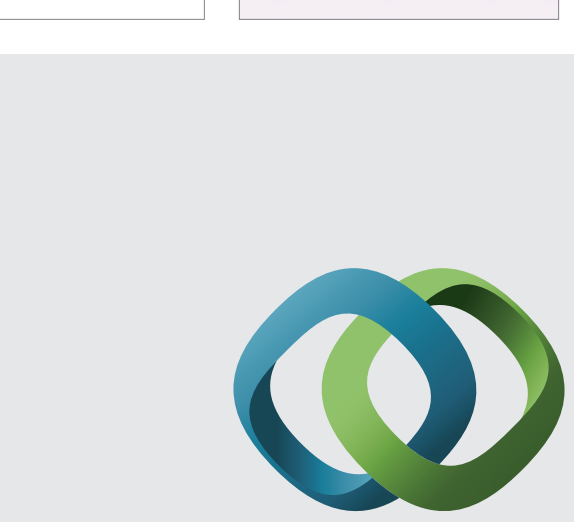

\section{Hindawi}

Submit your manuscripts at

http://www.hindawi.com
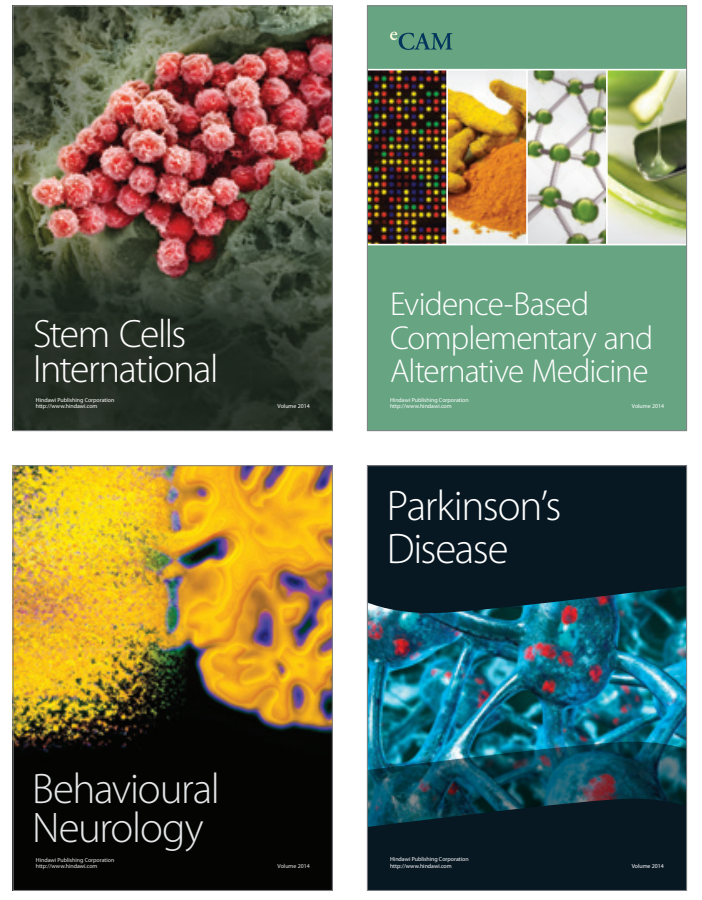
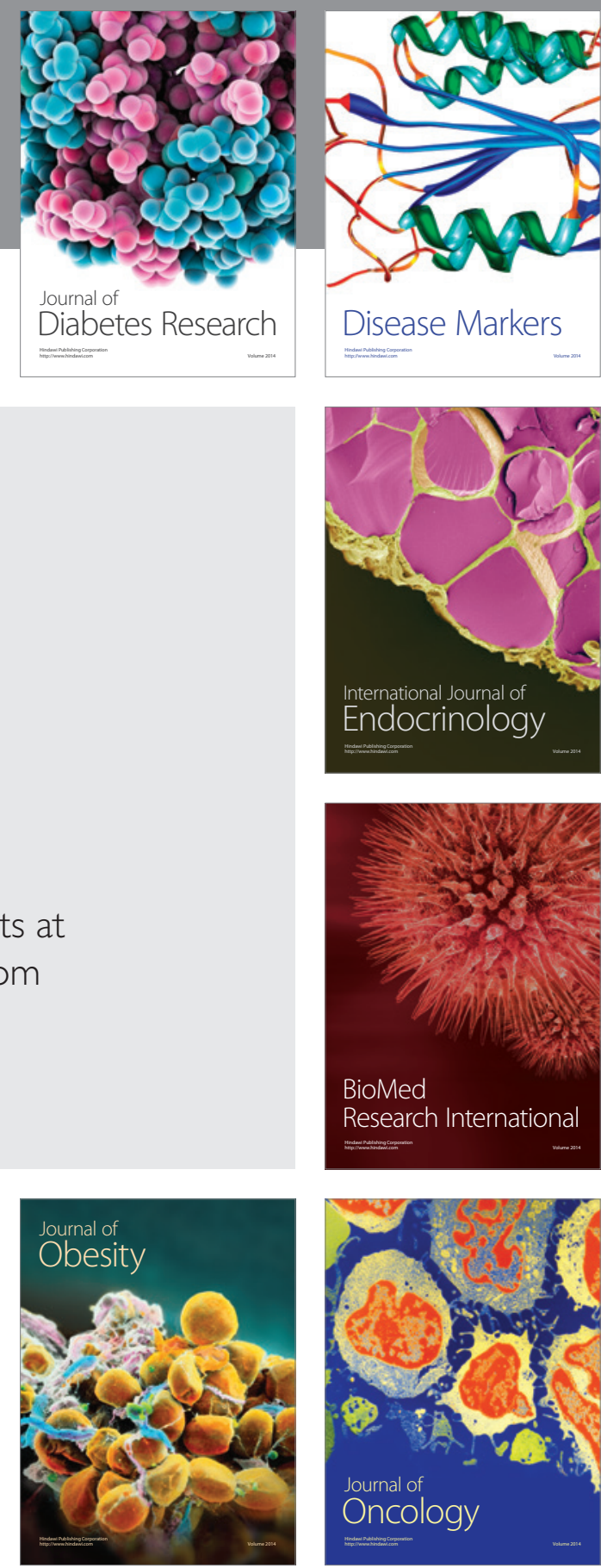

Disease Markers
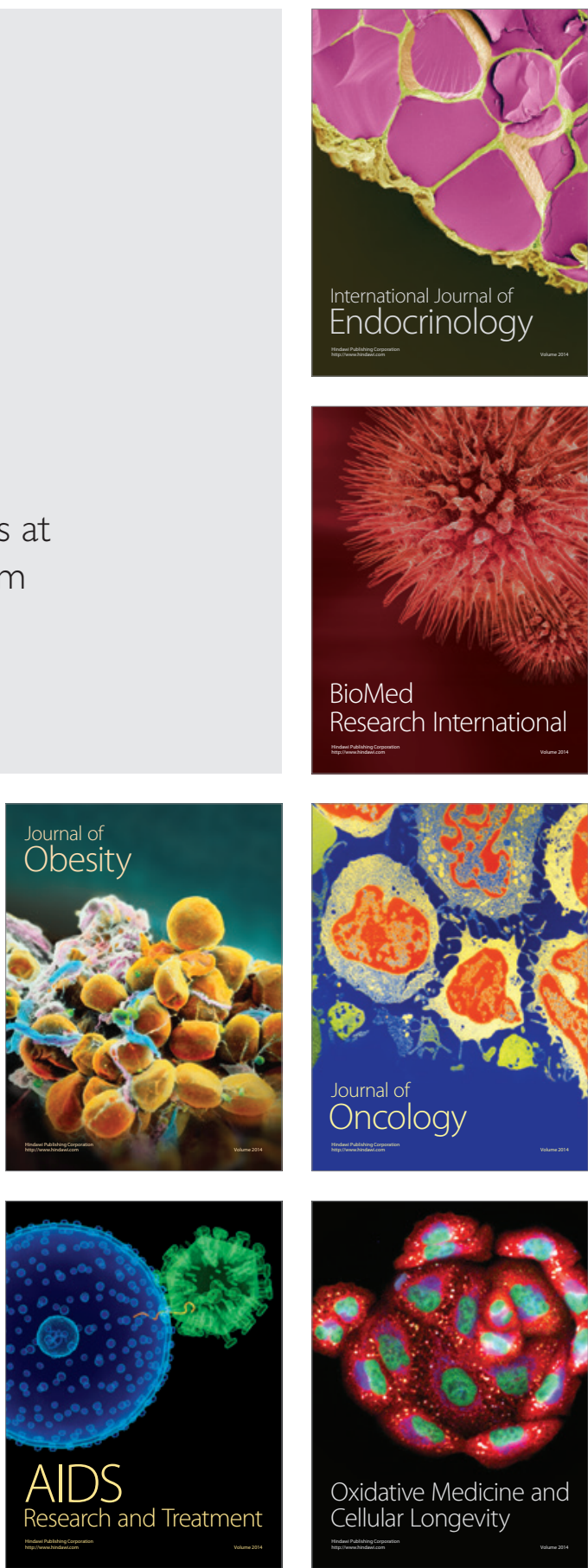